Chirurg 2021 · 92:573

https://doi.org/10.1007/s00104-021-01413-w Angenommen: 26. März 2021

Online publiziert: 22. April 2021

(c) Springer Medizin Verlag GmbH, ein Teil von Springer Nature 2021

\section{Originalpublikation}

Qentué F, Elias D, Roca L et al (2021) Cytoreductive surgery plus hyperthermic intraperitoneal chemotherapy versus cytoreductive surgery alone for colorectal peritoneal metastases (PRODIGE 7): a multicentre, randomised, open-label, phase 3 trial. Lancet Oncol 22:256-266

Hintergrund. Peritoneale Metastasen beim Kolorektalkarzinom (KRK) gehen mit einem reduzierten Gesamtüberleben und einer schlechteren Prognose einher als extraperitoneale Metastasen. In der französischen Studie wurde der Stellenwert der hyperthermen intraperitonealen Chemoperfusion (HIPEC) bei Patienten mit resektabler Peritonealkarzinose nach zytoreduktiver Chirurgie (CRS) untersucht.

Material und Methoden. In der prospektiv randomisierten Prodige-7-Studie wurden an 17 französischen Krebszentren Patienten zwischen 18 und 70 Jahren mit einem peritoneal metastasierten KRK und einem Peritonealkarzinosein$\operatorname{dex}(\mathrm{PCI}) \leq 25$ rekrutiert. Patienten, die eine CRS mit kompletter makroskopischer Resektion erhielten, wurden in zwei Arme randomisiert: CRS allein vs. CRS plus HIPEC mit Oxaliplatin. Primärer Endpunkt war das Gesamtüberleben. Sekundäre Endpunkte waren die postoperative Morbidität nach 30 und 60 Tagen.

M. Anthuber · A. Straßmüller

Klinik für Allgemein-, Viszeral- Transplantationschirurgie, Klinikum Augsburg, Augsburg, Deutschland

\title{
Ist die hypertherme intraperi- toneale Chemoperfusion (HIPEC) nach zytoreduktiver Chirurgie beim peritoneal metastasierten Kolorektalkarzinom entbehrlich?
}

Ergebnisse. Zwischen 2008 und 2014 wurden 265 Patienten in die Studie eingeschlossen. 132 Patienten wurden in den CRS-Arm und 133 Patienten in den CRS + HIPEC-Arm randomisiert. Nach 64 Monaten Follow-up zeigte sich kein signifikanter Unterschied hinsichtlich des Gesamtüberlebens (41,2 Monate im CRS-Arm und 41,7 Monate im CRS + HIPEC-Arm). In einer Subgruppenanalyse zeigte sich jedoch ein Überlebensvorteil für Patienten mit einem PCI von 11-15 in der CRS + HIPECGruppe. Nach 30 Tagen verstarben in beiden Gruppen je 2 Patienten (Letalität $=2 \%)$. Die Morbidität mit schweren Komplikationen (Grad 3-5 nach Common Terminology Criteria of Adverse Events [CTCAE]) war nach 30 Tagen in beiden Gruppen ähnlich (56 [42\%] von 133 in der CRS + HIPEC-Gruppe vs. 42 [32\%] von 132 in der CRS-Gruppe; $p=0,083)$. Nach 60 Tagen fand sich dagegen in der CRS + HIPEC-Gruppe eine signifikant höhere Morbiditätsrate (34 [26\%] von 131 vs. 20 [15\%] von 130 ; $p=0,035)$.

\section{Kommentar}

Mit 41 Monaten war das mediane Gesamtüberleben durch die alleinige CRS fast zweimal so hoch wie in einer 2003 publizierten holländischen Studie [1]. Die Daten verdeutlichen jedoch, dass diese komplexe Chirurgie an zertifizierten Zentren erfolgen sollte, um die Letalität und die Komplikationsrate möglichst gering zu halten. Kritisiert wurde die Studie bereits vor der Publikation durch die aus heutiger Sicht - viel zu optimistische Hypothese, dass die HIPEC das mediane Überleben von 30 auf 48 Monate verbessern könnte. Eine weitere Limitation besteht in der Anwendung von Oxaliplatin, welches zu hoch dosiert und zu kurz (30 min) verabreicht wurde. Im Gegensatz zu Oxaliplatin konnten Verwaal et al. [1] einen deutlichen Überlebensvorteil mit Mitomycin $\mathrm{C}$ aufzeigen. Da sich in der Subgruppenanalyse ein Überlebensvorteil für Patienten mit einem PCI von 11-15 zeigte, sind weitere randomisierte kontrollierte Studien notwendig, um ein optimales risikoadjustiertes Therapieregime für diese Patienten zu finden.

\section{Korrespondenzadresse}

\section{Prof. Dr. M. Anthuber}

Klinik für Allgemein-, Viszeral- Transplantationschirurgie, Klinikum Augsburg Stenglinstr. 2, 86156 Augsburg, Deutschland matthias.anthuber@klinikum-augsburg.de

Interessenkonflikt. M. Anthuber und A. Straßmüller geben an, dass kein Interessenkonflikt besteht.

\section{Literatur}

1. Verwaal VJ, van Ruth S, de Bree E et al (2003) Randomized trial of cytoreduction and hyperthermic intraperitoneal chemotherapy versus systemic chemotherapy and palliative surgery in patients with peritoneal carcinomatosis of colorectal cancer. JClin Oncol 21:3737-3743 